Archives

$35 \mid 2005$

Indexer les exempla médiévaux

\title{
Trente ans de recherche sur les exempla
}

Jean-Claude Schmitt

\section{(2) OpenEdition \\ Journals}

\section{Édition électronique}

URL : http://journals.openedition.org/ccrh/3010

DOI : $10.4000 /$ ccrh.3010

ISSN : $1760-7906$

Éditeur

Centre de recherches historiques - EHESS

Édition imprimée

Date de publication : 10 janvier 2005

ISSN : 0990-9141

\section{Référence électronique}

Jean-Claude Schmitt, "Trente ans de recherche sur les exempla », Les Cahiers du Centre de Recherches Historiques [En ligne], 35 | 2005, mis en ligne le 01 juin 2011, consulté le 19 avril 2019. URL : http:// journals.openedition.org/ccrh/3010; DOI : 10.4000/ccrh.3010

Ce document a été généré automatiquement le 19 avril 2019

Article L.111-1 du Code de la propriété intellectuelle. 


\title{
Trente ans de recherche sur les exempla
}

\author{
Jean-Claude Schmitt
}

\section{Une enquête dans la durée}

1 Trente-cinq ans nous séparent de la parution de l'Index exemplorum de Frederic C. Tubach de $1969^{1}$, vingt-deux ans de celle du volume de la « Typologie des sources» de $1982^{2}$, qui a marqué une première étape des recherches collectives sur ce type de récit : c'est dire que notre rencontre nous donne le recul nécessaire pour mesurer l'apport des enquêtes et des publications héritières fidèles et bénéficiaires de ces premiers instruments de travail.

2 Au-delà de l'intérêt particulier qu'elle présente pour l'histoire propre de l'équipe du Groupe d'anthropologie historique (Gahom), organisatrice de cette rencontre, la date de 1982 marque sans nul doute un moment charnière dans l'étude des exempla en général. Il y a un «avant " du volume de la "Typologie » comme il y a un "après " de cette publication.

3 Avant, il y eut, bien sûr, l'Index de Tubach. Mais cet auteur et son équipe n'ont pu réaliser leur immense travail qu'en s'appuyant sur des éditions de recueils d'exempla antérieures, publiées depuis le XIXe siècle, et surtout sur l'œuvre incomparable de défrichement de l'abbé Jean Thiebaut Welter. Car s'il y a une date à retenir avant celle de 1969, c'est bien, quelque quarante ans plus tôt, celle de 1927, année de la parution de L'Exemplum dans la littérature religieuse et didactique du Moyen Âge, qui a fait l'objet d'un reprint en 1973. Mais remontons plus haut encore de cinquante ans, jusqu'à l'admirable édition d'Albert Lecoy de la Marche, Anecdotes historiques, légendes et apologues tirés du recueil inédit d'Étienne de la Marche (1877), qui commence maintenant seulement à être remplacée par une édition critique complète et conforme à nos critères scientifiques. À cette édition, associons, quelques années plus tard, celle, également partielle malheureusement, de Thomas Frederic Crane, The Exempla or Illustrative Stories from the Sermones vulgares of Jacques de Vitry (1890, reprint 1967). 
4 L'intérêt pour les exempla est donc ancien: il a joui d'un premier élan à partir de la deuxième moitié $\mathrm{du}$ XIX ${ }^{e}$ siècle, qui s'est traduit par des éditions de textes toujours très utiles (Lecoy de la Marche, Crane, Strange pour Césaire de Heisterbach) et des catalogues de manuscrits, depuis celui de Barthélemy Hauréau (pour les manuscrits de la Bibliothèque nationale et des bibliothèques françaises) jusqu'à J. A. Herbert (pour ceux de la Bristish Library) ${ }^{3}$. L'intérêt renaît dans les années soixante, soixante-dix du Xxe siècle, à la faveur de l'orientation des études historiques et ethnologiques vers les questions de l'historicité du folklore, des traditions orales, des « récits brefs » : il faut citer ici aussi bien la collection des «Folklore Fellows Communications » - où Thomas Frederic Crane avait déjà été publié et qui a accueilli bien plus tard l'Index de Tubach - que les recherches de narratologie illustrées entre autres par Lüthi et Jolles ${ }^{4}$ et surtout par la Morphologie $d u$ conte de Vladimir Propp ${ }^{5}$; c'est à l'intersection de ces courants et en les faisant se rejoindre que se situent le travail de Jacques Le Goff et l'enquête collective à laquelle il a donné une impulsion première et décisive, et enfin le volume de la «Typologie » de 1982, où se croisent des préoccupations historiques et un essai d'analyse structurale dû à Claude Bremond.

5 En 1982, donc, la recherche rebondit, profitant d'une définition du genre tenant compte habilement des caractéristiques formelles du récit bref et de son "fonctionnement " particulier dans un contexte social et idéologique précis, soucieux de préciser sa fonction didactique, attentif à voir en lui, au delà des effets d'imposition d'une leçon morale et religieuse, le lieu d'un échange culturel entre clercs et laïcs.

6 À la suite de ces premières propositions, le volume de 1992 sur Les Exempla médiévaux a offert une précieuse "Introduction à la recherche » qui présente un état des travaux menés jusqu'à cette date, une bibliographie et surtout un index de l'Index de Tubach - le « Tubach à l'envers »- permettant au chercheur de ne plus partir seulement des « types » narratifs résumés et nommés par cet auteur - mais des exempla originaux, correspondants à ces "types» et se trouvant attestés dans une quinzaine de recueils médiévaux. Ce renversement de perspective a permis de constater que les dépouillements de recueils annoncés par Tubach n'étaient pas exhaustifs et que l'Index comportait nombre d'erreurs, ce qui n'enlève rien, naturellement, aux immenses mérites de cette œuvre pionnière.

7 Troisième étape, en 1998, à la faveur d'un colloque sur Les Exempla médiévaux: nouvelles perspectives. Son caractère international montre d'emblée l'ouverture à d'autres équipes qui se sont lancées sur les brisées du groupe parisien en Italie, en Espagne, en Allemagne, en Hongrie. Ouverture interdisciplinaire aussi, puisque l'histoire littéraire (on pense ici à Jean-Yves Tillette et au regretté Jacques Monfrin) fait bon ménage avec l'histoire de la prédication. L'enrichissement par rapport aux débuts de l'enquête est remarquable, en particulier par la découverte d'une géographie médiévale des exempla qui apparaissait mal jusque-là.

8 Définition, repérage, classification : à ces premières tâches, menées pendant vingt ans à partir de 1982, d'autres se sont ajoutées en chemin: les unes ont porté sur la traduction de choix d'exempla au profit d'un public plus vaste, tel Prêcher d'exemples de $1985^{6}$. D'autres sur l'édition critique de recueils jusqu'à présent peu disponibles parce que n'existant que sous forme manuscrite ou dans des éditions incunables ou anciennes ou au mieux dans des éditions non scientifiques : la Scala Coeli de Jean Gobi a été parfaitement éditée par Marie-Anne Polo de Beaulieu en 1991, un premier volume de la monumentale édition critique du recueil d'Étienne de Bourbon est paru en 2003, celle de l'Alphabetum 
narrationum est annoncée. À ces travaux fondamentaux se sont joints des recherches visant plutôt à l'interprétation historique de certains dossiers, qu'il s'agisse des rapports entre culture savante et culture « populaire » (à propos, dès 1979, du Saint Lévrier ${ }^{7}$ ), de la place des animaux dans les exempla [L'animal exemplaire, 1999], ou plus généralement de la prédication avec les travaux de Nicole Bériou ${ }^{8}$, de Carlo Delcorno ${ }^{9}$, de David d'Avray ${ }^{10}$, de Rosa Maria Dessi et Michel Lauwers ${ }^{11}$, une part de ces chercheurs en retrouvant avec d'autres dans le projet d'édition des Sermons de Jacques de Voragine ${ }^{12}$.

\section{La forme narrative}

9 Après ce rappel d'une recherche foisonnante qui mobilise les énergies depuis plus de vingt ans, tentons de dégager quelques axes de réflexions qu'il conviendrait de privilégier dans les années à venir. Les premières questions importantes concernent les caractéristiques formelles des exempla.

10 L'exemplum répond à une définition complexe [L'Exemplum, 1982, p. 27-38], qui doit à la fois tenir compte de sa nature de récit, de sa brièveté, de son paradoxe d'histoire souvent fictive, mais vraisemblable et donnée pour « authentique ». Ce récit se prévaut en effet d'une " autorité », c'est-à-dire d'une référence à un personnage "digne de foi » ou à un écrit passé qui « autorise ", garantit l'« authenticité » du dire. C'est un récit que nous ne connaissons que sous une forme écrite, mais qui se situe fréquemment en amont et en aval dans une chaîne de transmission orale. C'est un texte enchâssé dans un texte plus vaste (en premier lieu un sermon) et même un ouvrage (un recueil d'exempla). Enfin, c'est un récit qui a une finalité idéologique, morale et religieuse le plus souvent, puisque l' exemplum se veut d'abord « exemplaire »...

11 À cerner simplement les conditions d'une définition d'un tel «genre » on voit surgir de nombreux thèmes de réflexion qui pourraient être retenus pour l'avenir :

12 En premier lieu, la question de l'oral et de l'écrit et de leur combinaison dans les exempla: comment l'expression orale affecte-t-elle la forme écrite, où transparaissent par exemple des tournures, une syntaxe, voire des mots de la langue vulgaire ; «oralité » de l'amont, mais aussi de l'aval, car l'exemplum a vocation à être dit, prêché, plutôt qu'à être lu. À cet égard, il faut se garder d'une assimilation de l'oral au "vulgaire ", même si les première recherches, dans la continuité de Anita Aarne-Thompson et de Frederic C. Tubach, ont surtout conduit à tenter de repérer, non sans raison, dans ces récits les traces de traditions orales «populaires » ou «folkloriques ». En amont, la chaîne des témoignages cités se veut, doit être savante, cléricale, car elle seule est "autorisée", mais fréquemment elle invoque la transmission orale, le bouche à oreille, qui n'est pas le monopole du "peuple», qui peut en provenir, mais à travers la médiation cléricale. En aval, le prédicateur use de l'exemplum selon les fins qu'ils poursuit: certes, il va à la rencontre des fidèles, les auditeurs du sermon, qui forment bien ce que l'Église nomme le "peuple », et il est souhaitable qu'il se mette à leur écoute, mais sa volonté est bien de leur transmettre et même de leur imposer les normes de la croyance et de la pratique du bon chrétien tel que l'entendent les clercs. L'opposition «écrit/oral» n'est pas superposable à l'opposition « savant/populaire » et du reste la cristallisation de ces deux couples méconnaît largement la complexité des problèmes en cause. Pour sa part, l' exemplum est le lieu d'une circulation de paroles, de motifs et de modèles divers, voire 
contradictoires, d'un pôle à l'autre, son caractère «métis » tenant à son usage même, à son insertion dans une parole cléricale destinée aux laïcs.

Qui parle dans les exempla? Il faudrait réfléchir davantage à la mise en scène de l'énonciation et de l'« énonciateur » : comment sont nommés les témoins et garants de la parole de vérité que l'exemplum prétend être ? Où se situe le "je» de l'«auteur", collecteur d'histoires, qu'il soit actif et dise audivi, legi (j'ai entendu, j'ai vu) ou qu'il soit passif et se contente d'un legitur (on lit)? Dans les nombreux travaux et colloques portant actuellement sur «l'individu » et la "subjectivité », il y aurait intérêt à ce que de telles questions soient posées à propos de l'exemplum, comme cela a été fait déjà à propos de la notion d'«auteur $»^{13}$. On sait que le prédicateur et même le compilateur d'exempla, à l'instar des « auteurs » médiévaux, n'invente rien qui ne soit déjà là. L'importance de la sélection de la «matière prédicable » et en particulier des exempla, l'agencement de ceuxci, l'adaptation à un contexte à chaque fois différent - qu'on entende par là un texte : le recueil thématique ou alphabétique, le sermon, ou bien un public d'auditeurs - . Le prédicateur ne cesse de faire des choix, de tracer un parcours intellectuel singulier qu'il faut tenter de reconstituer. Les moralisations à la fin des récits et les entrées multiples des exempla signalées dans les recueils alphabétiques par un " hoc valet etiam ad " (cela s'applique également à) permettent de refaire au moins un bout du chemin du prédicateur, de comprendre ce qui a pu justifier pour lui la mobilisation de tel récit plutôt que de tel autre et le rapprochement de deux ou trois récits apparemment hétérogènes : la présence d'un mot peut suffire, ou bien l'évocation d'un personnage, d'un type d'action, une simple image.

Un trait spécifique de l'environnement de l'exemplum est sa double contextualisation (au sens étroit du texte qui l'englobe) dans un recueil et, plus rarement, dans un sermon. Dans le meilleur des cas, nous retrouvons le même exemplum non seulement dans des recueils différents, mais aussi dans un ou plusieurs sermons. Une telle situation est particulièrement précieuse : le sermon qui contient des exempla a été noté en «aval » de la prédication effective (pour reprendre ici la terminologie de Michel Zink). Certes, il faut se garder d'y voir une trace immédiate de la parole vive du prédicateur: il nous est conservé en latin (alors que les sermons " au peuple » étaient nécessairement prononcés en langue vernaculaire) afin de servir de modèle à d'autres sermons à venir (c'est le cas des sermons de Jacques de Vitry, dans lesquels sont enchâssés des exempla). Tout de même, ces sermons nous rapprochent de la " performance » réelle de la prédication, un peu plus en tout cas que les recueils, surtout les recueils alphabétiques, qui par comparaison font figure de "boîte à outils " du prédicateur. Quand les deux modes d'insertion sont attestés pour un même exemplum, il devient passionnant d'étudier ses transformations d'un contexte à l'autre: d'analyser les modes d'enchaînement de plusieurs exempla dans le sermon et le mode d'«accrochage» de chacun d'eux à un passage donné ; de vérifier si les thèmes au service desquels les exempla figurent dans les sermons sont bien ceux qu'ont prévus les renvois hoc valet etiam ad des recueils alphabétiques; d'apprécier l'amplification ou la réduction du récit, de sa forme "type " dans le recueil à sa forme « en acte » dans le sermon, et de voir quel élément a mérité la première et lequel a pâti de la seconde; d'être attentif aux substitutions de mots, de noms, de personnages et même d'épilogues, quand le même exemplum s'achève une fois sur un beau mariage, une autre fois sur la pénitence et l'entrée au couvent... Une comparaison systématique de toutes les versions d'un seul exemplum abondamment cité et 
suivant un axe chronologique long peut être riche d'enseignements sur la diversité et l'évolution du cadre historique de ses usages ${ }^{14}$.

\section{Les contenus}

L'exemplum peut apparaître à bon droit comme un « miroir » de la société médiévale, mais certainement pas au sens du Speculum d'un Vincent de Beauvais : il ne hiérarchise pas les catégories et les êtres, il raconte et entraîne dans le flux continu de sa narration les hommes et les animaux, les démons et les anges, les grandeurs et les faiblesses de la vie quotidienne. S'il participe sans doute de «l'encyclopédisme » du XIII siècle ${ }^{15}$, il le fait à sa manière, celle d'une « encyclopédie narrative » où tout est dit, mais sur le mode du récit continu, souvent facétieux et critique, par des images et des mises en scène frappantes, " exemplaires ", un peu à la manière des caricatures de Plantu... Rien n'échappe a priori à l'œil avisé de l'exemplum : ni la diversité des êtres (hommes, animaux, êtres surnaturels, l'humanité étant pensée à la lumière de ceux qui se distinguent d'elles), les lieux (les églises, les cimetières, la ville, les châteaux, les chemins, la mer, l'au-delà sous toutes ses formes), les temps (la nuit et le jour, les heures canoniques, les dimanches et jours de fête), les statuts sociaux (clercs, moines, nobles, marchands, paysans, hommes et femmes, enfants, prostituées, usuriers, etc.), les activités pratiques et symboliques (prière, pèlerinage, messe, sommeil et rêve, repas, scènes de séduction, tournois, etc.). Autrement dit, les exempla sont un véritable trésor où l'on peut puiser à l'infini, un peu comme invitent à le faire, dans le domaine de l'iconographie, les Cantigas de Santa Maria d'Alphonse le Sage ${ }^{16}$. Et on ne s'est pas privé d'y puiser, comme en témoigne la liste des sujets de maitrise du genre «Le diable dans les « exempla », « La femme dans les exempla », "Les animaux - ou tel animal - dans les "exempla ». Ces travaux sont bien sûr légitimes, dans les limites du genre académique qui est le leur. Il ne faudrait cependant pas les multiplier au risque de perdre de vue la cohérence formelle et sémantique de l'ensemble de ces récits, dans les contextes variés qui sont les leurs. C'est ce contexte, répétons le, qui décide des choix du prédicateur, celui par exemple de parler ici d'animaux sur un mode qui s'apparente davantage à la fable, et là d'hommes ordinaires. Le parallèle avec les Cantigas s'applique parfaitement: dans ces derniers aussi, on peut tout trouver en image, mais c'est justement le fait qu'il s'agisse de narrations en images, caractérisées par un mode de figuration spécifique, qui est intéressant et c'est cela qu'il faut étudier avant tout objet particulier.

\section{Les fonctions}

16 Pour les prédicateurs, le but des exempla est de donner une "leçon morale » à leurs auditeurs. L'exemplum est par cela même le lieu d'une relation hiérarchique (le prédicateur ayant tous les avantages du locuteur et du "donneur de leçon») et idéologique (par le contenu du récit et surtout sa moralisation). Dans l'exemplum, un supérieur (le maître des novices qu'est Césaire de Heisterbach, le prédicateur séculier ou plus souvent Mendiant) parle à des inférieurs (respectivement les novices ou les auditeurs laïcs du sermon). Mais cette dimension hiérarchique est assez largement occultée par le caractère universaliste de la langue des exempla, qui semble s'adresser à tout le monde et niveler assez largement les différences sociales dans un inventaire pittoresque de tous les petits et plus gros vices de la gent humaine. Le rapport hiérarchique et la contrainte 
idéologique se cachent derrière la tendance " démocratique " du christianisme que partage largement l'exemplum.

Un autre trait important de l'exemplum est qu'il doit favoriser une forme de conversio, mais de conversion interne à la chrétienté : il n'est destiné ni aux juifs, ni aux musulmans. Tout au plus aux hérétiques, encore que leur présence abondante dans les récits ne prouve pas qu'ils en fussent les destinataires. Ceux-ci sont les chrétiens ordinaires, qu'il s'agit de convaincre tout à la fois de leur imperfection foncière et de leur nature perfectible : avec l'exemplum (comme avec le purgatoire, et ce rapprochement n'est pas fortuit puisque tous deux appartiennent à la même phase de l'histoire de la chrétienté), l'espoir est toujours permis. En tout cas, le prédicateur n'est pas un missionnaire, et c'est pourquoi il peut faire usage des exempla, qui supposent une connivence entre locuteurs et destinataires qui ne peut exister dans le cas d'une tentative de conversion « externe ». Prédicateurs et laïcs appartiennent au même monde, partagent la même foi, les mêmes valeurs, se reconnaissent les mêmes travers et savent en rire ensemble.

Ce qui m'amène à parler de la question de la réception, plus difficile à résoudre malheureusement que celle de l'émission: quels furent les effets des exempla, dans l'instant (il est parfois fait mention d'une réaction de l'auditoire, mais c'est bien rare) et à plus long terme : l'efficacité postulée s'est-elle vérifiée, sur les usuriers, les adultères, les femmes fardées ? La répétition des mêmes histoires entre le XIII et le XVe siècle au moins pourrait suggérer une réponse négative. Mais il faut tenir compte, autant que de la lenteur de l'évolution de la morale, des inerties de la culture cléricale et lettrée: les prédicateurs ont perfectionné le système de classification, rendu plus pratique et rapide, grâce à des renvois et des index, la recherche des exempla utiles à tel ou tel sujet, amplifié peu à peu la masse de récits disponibles, privé les récits des références trop particulières ou locales qui auraient pu nuire à leur application universelle, mais ils n'ont pas vraiment renouvelé le genre pendant toute la période. Encore faudrait-il analyser de plus près les effets sur ce corpus inflationniste de la succession des types de locuteurs (cisterciens, séculiers, franciscains, dominicains), des changements intervenus dans la situation générale de l'Église (au temps du Schisme par exemple) et des différences nationales de plus en plus marquées dans une chrétienté qui gardait néanmoins son unité culturelle, au-delà des péripéties du Schisme.

Les questions que je pose ici vont bien sûr au-delà du propos de cette journée consacrée aux aspects techniques de la recherche. Mais elles sont de celles qui doivent sous-tendre toutes les étapes de la recherche et guider celle-ci. L'indexation, la constitution de banques de données sont au service d'un projet intellectuel, elles n'ont pas de fin en soi. Mais en même temps, il n'y a pas de relation univoque entre conceptualisation et travail technique : celui-ci produit heureusement un effet de feed-back sur la définition des problématiques, donnant à chaque fois des impulsions nouvelles à la recherche. 


\section{NOTES}

1. Frederic C. Tubach, Index exemplorum, A Handbook of Medieval Religious Tales, Helsinki, 1969 (FFC 204).

2. Claude Brémond, Jacques Le Goff, Jean-Claude Schmitt, L'« Exemplum », Turnhout, Brepols, 1982, Typologie des sources du Moyen Âge, fasc. 40, 2e éd. augmentée par Jacques Berlioz, 1996.

3. Barthélemy Hauréau, Notices et extraits de quelques manuscrits latins de la Bibliothèque nationale, Paris, 1890-1893 ; J. A. Herbert, Catalogue of Romances in the Department of Manuscripts in the British Museum, vol. 3, Londres, 1910.

4. André Jolles,Einfache Formen. Legende / Sage / Mythe / Rätsel / Spruch / Kasus / Memorabile / Märchen / Witz., Halle, 1930; 2. Aufl., Halle, 1956 ; 3. Aufl., Tübingen, 1965 ; id., Formes simples, Paris, Le Seuil, 1972, trad. fr.

5. Vladimir Propp, Evgueni Mélétinski, Morphologie du conte, Paris, Le Seuil, 1970, trad. fr.

6. Jean-Claude Schmitt (s. d.), Prêcher d'exemples. Récits de prédicateurs du Moyen Âge, Paris, Stock/ Moyen Âge, Paris, 1985 ; Jacques Berlioz, Claude Brémond, Catherine Vallantin, Formes médiévales du conte merveilleux, Paris, 1989.

7. Jean-Claude Schmitt, Le Saint Lévrier. Guinefort guérisseur d'enfants depuis le XIII' siècle, Paris, Flammarion, 1979. $2^{\mathrm{e}}$ édition augmentée, 2004.

8. Nicole Bériou, Les Maîtres de la parole. La prédication à Paris au XIII siècle, Paris, Institut d'Études augustiniennes, 1998 ; Ead., Sermons et visites pastorales de Federico Visconti, archevêque de Pise, Rome, École française de Rome, 2001.

9. Carlo Delcorno, « La predicazione volgare in Italia (sec. XIII-XIV). Teoria, produzione, ricezione ", Revue Mabillon, n. s., 4, t. 65, 1993, p. 107 ;id., « Pour une histoire de l'exemplum en Italie ", in Jacques Berlioz et Marie Anne Polo de Beaulieu (dir.), Les Exempla médiévaux : nouvelles perspectives, Paris, Champion, Nouvelle bibliothèque du Moyen Âge, 47, 1998, spéc. p. 147-176.

10. David d'Avray, The Preaching of the Friars: Sermons Diffused from Paris Before 1300, Oxford, Clarendon Press, 1985 ; The Death of the Prince: Memorial Preaching Before 1350, Oxford, Clarendon Press, 1994 ; en collaboration avec Nicole Bériou, Modern Questions about Medieval Sermons : Essays on Marriage, Death, History and Sanctity, Spolète, Centro italiano di studi sull'alto medioevo, 1994.

11. Michel Lauwers, Rosa Maria Dessi, La Parole du prédicateur, Nice, collection du CEM, 1997.

12. "Le Thésaurus de sermons de Jacques de Voragine on line » : base de données collaborative dirigée par Nicole Bériou, Lyon 2, Projet international présenté sur le site de l'ISH de Lyon : «La communication de masse au Moyen Âge. Jacques de Voragine et ses sermons. Vulgariser et éduquer par la parole » dans Les dossiers, janvier 2001, nº 17 [www.ish-lyon.cnrs.fr].

13. Marie Anne Polo de Beaulieu, «L'émergence de l'auteur et son rapport à l'autorité dans les recueils d'exempla», in Michel Zimmermann et alii, éd., Auctor et auctoritas. Invention et conformisme dans l'écriture médiévale, Université de Versailles, Saint-Quentin-en-Yvelines, 11-13 juin 1999, Paris, École des chartes, 2001, Mémoires et documents de l'École des chartes, 59, p. 175-200.

14. J'en ai fait l'expérience dans l'étude (inédite) de l'exemplum, «L'ange et l'ermite », connu au moins depuis le début du XIII' siècle et jusqu'au XX ${ }^{e}$ siècle.

15. Jacques Berlioz et Marie Anne Polo de Beaulieu, «Les recueils d'exempla et la diffusion de l'encyclopédisme médiéval ", in L'Enciclopedismo medievale, actes du colloque de San Giminiano, 6 -10 octobre 1992, a cura di Michelangelo Picone, Ravenne, Longo, 1994, p. 179-212.

16. José Filgueira Valverde (éd.), Cantigas de Santa Maria, Madrid, Castalia ed., 1985. 


\section{AUTEUR}

JEAN-CLAUDE SCHMITT

EHESS/CRH/GAHOM 